\title{
Les magasins d'usine : lieux commerciaux ou éléments de valorisation du patrimoine d'espaces urbains en reconversion?
}

Warehouses : trading places or components of the development heritage of reconverted urban spaces?

Factory Outlet Center: Handelsstandorte oder Elemente der Inwertsetzung des Erbes von im Umbau befindlichen Stadtvierteln?

\section{Nicolas Lebrun}

\section{OpenEdition}

\section{Journals}

Édition électronique

URL : http://journals.openedition.org/rge/1483

DOI : $10.4000 /$ rge. 1483

ISSN : 2108-6478

Éditeur

Association des géographes de l'Est

Édition imprimée

Date de publication : 1 juin 2006

ISSN : 0035-3213

\section{Référence électronique}

Nicolas Lebrun, «Les magasins d'usine : lieux commerciaux ou éléments de valorisation du patrimoine d'espaces urbains en reconversion? », Revue Géographique de l'Est [En ligne], vol. 46 / 3-4 I 2006, mis en ligne le 21 décembre 2009, consulté le 08 septembre 2020. URL : http:// journals.openedition.org/rge/1483 ; DOI : https://doi.org/10.4000/rge.1483

Ce document a été généré automatiquement le 8 septembre 2020.

Tous droits réservés 


\title{
Les magasins d'usine : lieux commerciaux ou éléments de valorisation du patrimoine d'espaces urbains en reconversion?
}

\author{
Warehouses : trading places or components of the development heritage of \\ reconverted urban spaces?
}

Factory Outlet Center: Handelsstandorte oder Elemente der Inwertsetzung des Erbes von im Umbau befindlichen Stadtvierteln?

Nicolas Lebrun

\section{Introduction}

1 Les villes industrielles, confrontées à des problèmes de reconversion ont cherché à promouvoir de nouvelles activités. A cette fin, elles ont souvent développé des projets d'aménagement commercial en valorisant leur patrimoine industriel. Cet article propose de s'intéresser plus spécifiquement au rôle des magasins d'usine dans l'effort nécessaire de renouvellement urbain et de maintien de l'identité patrimoniale des villes industrielles en crise (Paris, 2001).

2 Aujourd'hui, les lieux de localisation de la production industrielle ont changé, l'origine des articles vendus dans les magasins d'usine aussi et pourtant les localisations marchandes associées à ces productions demeurent voire se renforcent.

Deux exemples français de sites industriels textiles autrefois florissants doivent être évoqués :

1. La ville de Troyes, «capitale de la maille» est aujourd'hui le premier site français de magasins d'usine.

2. La ville de Roubaix, n'a jamais eu autant de magasins d'usine que depuis que la production textile y a quasiment disparu. 
Ce n'est pas un hasard si la localisation des principales concentrations de magasins d'usine coïncide avec des espaces à dominante industrielle, notamment textile. En effet, le magasin d'usine, du moins à l'origine, a vocation à proposer au consommateur des articles produits sur place. Le consommateur y voyait le moyen d'acquérir à prix modéré des articles dont il connaissait la provenance et la qualité. Magasin d'usine est, tout à la fois synonyme d'affaires pour le client, puisque celui-ci s'épargne les marges des circuits de distribution classique court-circuitant les filières d'écoulement traditionnelles, et gage de sécurité, un article produit à proximité paraissant toujours de meilleure qualité...

5 L'évolution du commerce d'usine n'a pas suivi le même sort que les sites industriels qui l'alimentaient. On remarque même, un renforcement de l'offre marchande de ce type dans des lieux frappés de plein fouet par les délocalisations industrielles.

\section{L'Évolution du fonctionnement des commerces d'usines}

6 L'évolution du concept de magasin d'usine a changé au fur et à mesure de l'évolution de la compétitivité des industries du pays d'une part, et, de l'évolution de la grande distribution.

\section{A. Évolution du concept : du magasin d'usine au magasin de déstockage}

7 Les relocalisations industrielles, fréquentes depuis le début des années 1980 dans le secteur textile, éloignent lieux de production et lieux de consommation. Elles rendent de ce fait obsolètes les magasins d'usines traditionnels intégrés au site de production. Par conséquent l'offre marchande proposée par les centres de magasins d'usine n'est plus directement liée aux productions locales mais répond davantage à des logiques de distribution globalisées. Dès lors, à la logique de proximité se substitue une logique de mondialisation qui se manifeste d'abord par le jeu d'enseignes. De fait, on trouvera les mêmes produits dans tous les magasins d'usine d'une même marque, ou d'un même circuit de distribution. Ces articles seront nécessairement différents des circuits de distribution classique, pour que ces derniers soumis à des tarifications moins avantageuses puissent garder le privilège d'écouler des nouveautés et des articles spécifiques. C'est un changement radical puisque du temps où le magasin d'usine était directement lié à un site de production proche, son offre était unique puisqu'elle était le reflet des productions locales. En Europe, ce changement de concept s'amorce dans les années 1980 (Collectif, 2004). Désormais, on parlera davantage de commerce de déstockage (outlet shops) que de commerce d'usine (factory shops). 
Figure 1 : Positionnement du magasin d'usine dans le dispositif de production et de consommation

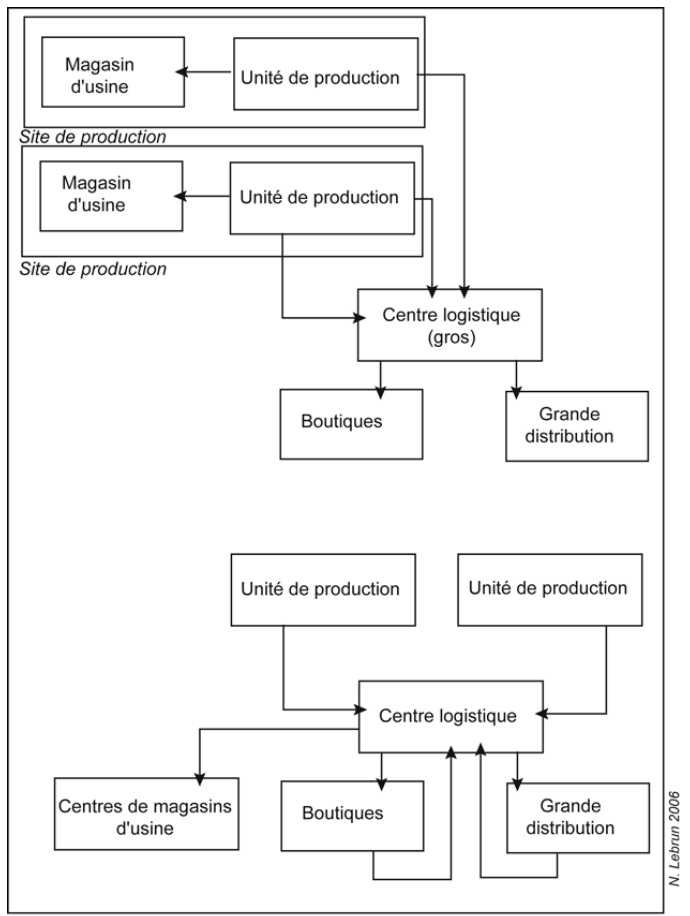

Positionnement du magasin d'usine dans le dispositif de production et de commercialisation En haut : système traditionnel

En bas : système modernisé

\section{B. Répercussions sur les formes commerciales}

8 Par ailleurs, l'évolution du secteur de la distribution s'accompagne d'une concentration des enseignes et des moyens logistiques mis en œuvre. De ce fait, le magasin d'usine isolé auprès d'un site de production semble moins viable qu'une concentration marchande spécialisée susceptible de drainer davantage de consommateurs.

Il en résulte qu'auparavant la proximité géographique entre des magasins d'usines s'expliquait par la concentration industrielle au sein d'une même ville ou d'une même région urbaine. Mais, l'implantation marchande n'étant qu'un corollaire de l'implantation industrielle, la localisation commerciale ne pouvait être affinée en fonction de prévisions de fréquentation. La concentration des magasins d'usines sur un nombre restreints de sites n'a pu se faire qu'à partir du moment où la dissociation entre activité de production et activité de distribution s'est manifestée. Cette réorganisation s'inscrit d'ailleurs dans une logique de concentration spatiale proche de celle de la grande distribution, que certains ont pu qualifier de post-fordiste, puisqu'elle reprend des éléments qui avaient fait le succès du déploiement industriel au XIX' siècle (Moati, 2001).

C'est pourquoi les premières concentrations de magasins d'usines ou de déstockage sont relativement récentes. L'intervention de grands groupes de distribution est elle aussi un phénomène nouveau. Certains de ces groupes sont chargés de gérer des sites de distribution sous une même enseigne (l'Américain Tanger Outlets ou encore le britannique Mc Arthur Glen), alors que d'autres sont chargés de veiller à l'écoulement d'articles de déstockage de plusieurs marques possédant des sites de productions communs (VF Outlet par exemple). Si cette immixtion du promoteur marchand dans la 
gestion des magasins d'usine s'est faite en France dès les années 1980, le concept a dû évoluer dans les années 1990 pour être viable, marquant le passage aux centres de magasins d'usine de seconde génération, qui excluent désormais les soldeurs et distributeurs classiques. Il est vrai que les centres de première génération ne connurent pas le succès escompté étant davantage des agrégats de commerces «bon marché » que de réelles concentrations de magasins d'usine : sur les 21 centres créés dans les années 1980, seuls quatre survécurent (Collectif, 2003).

11 Le fait que le commerce d'usine puisse faire l'objet de concentrations de commerces spécifiques permet en outre d'entrevoir la possibilité d'en faire un instrument de rénovation urbaine. Souvent les centres de déstockage s'installent dans des friches industrielles: c'est une opportunité pour les promoteurs de ces centres, mais aussi pour les aménageurs qui cherchent à réhabiliter ces espaces en crise par des opérations d'envergure. L'acteur politique et institutionnel et l'acteur économique se trouvent ainsi animés de motivations communes, ce qui a pu faciliter l'émergence de tels projets.

\section{L'image de la ville textile}

\section{A. Un espace de consommation existant}

Désormais, les localisations commerciales ne sont plus astreintes aux mêmes contraintes de localisation et répondent de ce fait davantage à des logiques de positionnement par rapport aux circuits de distributions traditionnels et par rapport aux foyers de consommateurs.

Or, la délocalisation des foyers de production n'a aucune incidence sur la localisation des foyers de consommation. C'est pourquoi, même si les sites industriels disparaissent, on observe une permanence dans la localisation des sites de magasins d'usine. Ce souci de garder une trace des sites de production passés témoigne aussi d'un attachement aux racines locales vivaces.

En effet, l'industrie textile française présentait cette caractéristique d'être fortement concentrée sur un plan spatial. Ainsi, une même ville pouvait comporter un nombre conséquent d'usines qui chacune avait son propre magasin d'usine. De fait, même lorsque les sites de productions étaient éparpillés dans la ville, ces espaces urbains se sont construits une image de villes des bonnes affaires due à l'essaimage des magasins d'usine au sein des quartiers industriels. Qui dit bonne image dit nécessairement creuset de consommateurs qui pouvaient venir de loin, non pas uniquement pour tel ou tel magasin, mais pour bénéficier des bienfaits de ladite concentration. C'est d'ailleurs une des caractéristiques du commerce vestimentaire que d'être beaucoup plus concentré que les autres formes marchandes (Bondue, 2000 ; Lebrun, 2002).

La disparition des sites industriels dans les villes textiles françaises s'est faite de façon progressive par le jeu des regroupements et des relocalisations. Pourtant, l'image textile des anciens gros foyers de production persiste, généralement associée à une image de qualité des marchandises fabriquées. La plupart des relocalisations se sont produites au bénéfice de pays moins développés sans tradition de savoir-faire particulière. Par conséquent, il aurait pu être dommage de remettre en cause les localisations des commerces, tant bien même la production aurait disparu. Il est vrai que le client n'est pas vraiment au courant des produits encore fabriqués localement aujourd'hui, et tant bien même le serait-il si le concept de bonnes affaires est reconduit, 
le consommateur peut encore y trouver son compte. Le temps est une valeur sûre, et s'appuyer sur l'image pérenne d'un savoir-faire ancien et entretenu est un bon instrument de marketing, à condition de ne pas s'enfermer dans l'immobilisme.

\section{B. La patrimonialisation du site de production textile}

16 Jouer de cette ambiguïté pour le promoteur marchand, c'est reconnaître l'existence d'une centralité-patrimoine (Lebrun, 2002), qui à défaut d'avoir un impact massif sur les habitudes et les déplacements, n'en demeure pas moins réelle. Mais accorder au temps une valeur dont la substance nous échappe, voilà un exercice auquel il est difficile de se résoudre. Alors on cherche à entretenir le vécu du lieu fonctionnel, on cherche à le renouveler. Longtemps dénigrée, l'image du site industriel ancien est conservée, fossilisée, voire recréée pour en exploiter les vertus. Ainsi, les usages du patrimoine se jouent parfois du temps: on pérennise l'instantané, on pastiche l'ancien pour donner l'illusion du temps, on crée une histoire à défaut de s'inscrire dans l'Histoire. Et de ce fait, le patrimoine a vite fait de devenir une valeur subjective... C'est ce que Laurent Devisme nous rappelle : «La patine du temps est aujourd'hui une valeur urbaine éminente qui n'a pas échappé à ceux dont la position dépend pour partie d'un prestige attribué par les autres. Ne pas être n'importe qui. Avoir du goût. Certaines centralités confêrent assurément ce que Pinçon et Pinçon-Charlot nomment une griffe spatiale par analogie avec la griffe d'un haut couturier» (Devisme, 1998).

\section{La patrimonialisation, élément créateur de nouvelles synergies}

17 La différenciation par le patrimoine peut somme toute être un moyen de redonner un second souffle à certains espaces fonctionnels « anciens » mis à mal par l'émergence de concurrences nouvelles. On peut qualifier ce type de recours au patrimoine de patrimonialisation défensive, puisqu'il s'agit de mettre en avant l'historicité et les valeurs patrimoniales du lieu afin de se démarquer dans un contexte de crise (Lebrun, 2002). C'est donc un moyen de résister à des espaces nouvellement créés, en entrée de ville par exemple, qui cherchent à s'appuyer sur des concepts commerciaux efficaces. Xavier Greffe nous rappelle ainsi que la dimension économique du patrimoine passe par une réflexion globale qui dépasse la simple référence au site, monuments et objets du patrimoine à conserver (Greffe, 2003). Il s'agit de raisonner en terme de «services patrimoniaux ", capables de procurer utilités et satisfaction et non de simplement réutiliser des lieux.

18 La différenciation par le patrimoine peut aussi être un moyen pour des espaces fonctionnels "sans passé " de s'accorder une légitimité vis-à-vis d'autres centres fonctionnels standardisés et sans âme. Nous parlons alors de patrimonialisation offensive (Lebrun, 2002), dans la mesure où il s'agit de court-circuiter l'effet temporel, et de créer un « effet patrimoine " pour des éléments fonctionnels récents peu après voire dès leur création. Il s'agirait alors d'actions visant à sauver les apparences en jetant le discrédit sur d'autres espaces anonymes et envahissants. Il peut s'agir néanmoins d'une réelle volonté patrimoniale et non d'une simple récupération abusive: l'extension de la notion de patrimoine répond ainsi à un souci réel comme le rappelle Maria GravariBarbas en affirmant que "Le patrimoine deviendrait alors un rempart contre l'espace vague et anomique» (Gravari-Barbas, 1996). Il s'agit en effet de lutter contre "avant tout la disqualification et l'homogénéisation de nos lieux de vies... Il suffit de penser à nos 
banlieues et aux disgracieuses zones commerciales et artisanales qui cernent le moindre de nos villages » (Augé, 1991).

Il est parfois possible, qu'en un même lieu, on retrouve des éléments de patrimonialisation défensive et offensive. C'est notamment le cas lorsque autour d'un reliquat patrimonial réinvesti, on cherche à développer une synergie propice à l'implantation d'activités nouvelles. Dans ce cas, plus que le patrimoine bâti, c'est l'imaginaire du lieu qui est réinvesti.

\section{Réinvestir les lieux par la réhabilitation et le renouvellement}

\section{A. La réhabilitation par le commerce, instrument de patrimonialisation défensive}

Les cas d'utilisation à des fins commerciales du patrimoine industriel bâti sont somme toute rares, puisqu'il s'agit toujours d'opérations ponctuelles et symboliques.

Parfois, il s'agit simplement de réutiliser un bâtiment industriel qu'il semblait regrettable de détruire. C'est alors un simple devoir patrimonial en rien motivé par un désir de réutiliser l'image industrielle du lieu. La mise en place d'un centre commercial (La Rotonde) dans une ancienne rotonde ferroviaire à Béthune, s'inscrit tout à fait dans cette logique.

Parfois on ne sauve que le nom, mais ce n'est pas là un usage propre aux utilisations purement marchandes : pensons aux multiples salles de spectacles qui réinvestissent les friches industrielles (par exemple La Laiterie à Strasbourg ou L'Usine puis La Cartonnerie à Reims).

Mais parfois, on observe une réelle continuité entre le passé du lieu et son utilisation actuelle. Continuité qui se manifeste d'une double façon : par un réinvestissement d'un lieu industriel sans dénaturation excessive et par une réappropriation de l'image industrielle. Or, seul le commerce dit d'usine, peut véritablement assurer cette continuité, tant bien même la disparition de l'activité industrielle et l'ouverture du site de magasin d'usine sont séparées de plusieurs années.

À ce titre, l'Usine, à Roubaix, est la première concentration de ce type tant par l'ancienneté que par la taille (Lebrun, 2004). Elle est un exemple saisissant de patrimonialisation défensive réussie. Ouvert en 1984, ce centre de magasins d'usine est situé dans un faubourg industriel. Il constitue un ensemble commercial intégré dans une ancienne usine textile restaurée, totalement dessaisie de sa fonction initiale, mais dont on a gardé tous les attributs industriels afin de souligner un lien de "parenté » de moins en moins évident. Tout est fait pour que la concentration de commerces soit perçue comme élément de l'industrie textile. Même la cheminée de l'usine est restée en place. A vrai dire, toute la ville de Roubaix cultive ce souvenir d'un passé industriel textile florissant. Le fait que se maintiennent sur Roubaix des sièges de chaînes vestimentaires (Camaïeu ou encore le vépéciste La Redoute), s'inscrit dans cette même logique. Mais si l'espace marchand de l'Usine affirme sa prise de relais d'une fonction autrefois développée, c'est bien sûr pour en récupérer les bénéfices. 
Photo 1 : Le centre commercial L'Usine de Roubaix

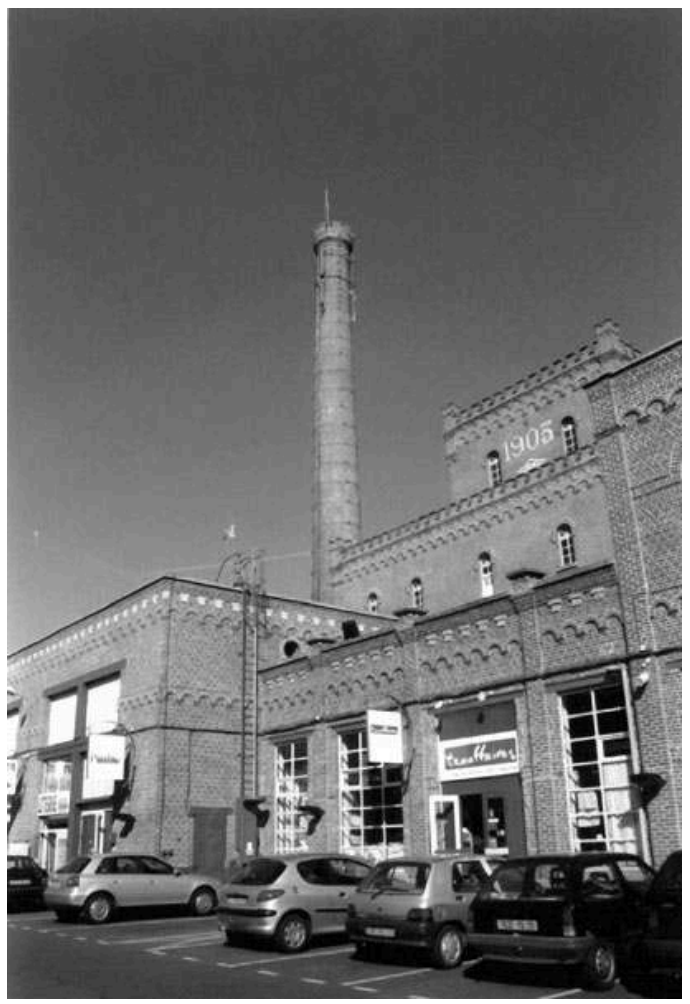

N. Lebrun, 2001

Mais suffit-il de se présenter comme dans la lignée d'une fonction urbaine pour être instrument de revitalisation urbaine, et entraîner une redynamisation des alentours ? À vrai dire, quand le commerce est instrument de patrimonialisation c'est plus pour son propre compte, dans la mesure où les espaces en friches sont généralement bien plus vastes. On remarque ainsi que si la fréquentation de Roubaix est en hausse, les retombées en terme d'aménagement des friches connexes à l'Usine sont quasi inexistantes. De ce fait, l'Usine, née d'une initiative isolée, demeure certes un exemple de réinvestissement par le commerce réussi, mais sans véritable portée urbanistique.

\section{B. Le renouvellement par le commerce, instrument de patrimonialisation offensive. Le cas des centres de magasins d'usine de la périphérie de Troyes.}

Le cas des magasins d'usines de la ville de Troyes s'inscrit dans une logique plus complexe :

1. La fonction industrielle textile est encore présente à l'état lacunaire ce qui permet de cultiver des éléments de patrimonialisation défensive mal valorisés (c'est-à-dire des petits commerces d'usine attenants à des sites de production)

2. L'essentiel des commerces d'usines se concentre dans deux centres commerciaux (Marques Avenue et Mc Arthur Glen) situés sur ou à proximité d'anciens sites industriels mais où bien peu de choses pourraient rappeler cette fonction originelle.

27 On observe donc une dissociation physique entre les lieux marchands d'aujourd'hui et les sites textiles industriels initiaux. Pourtant, un simple changement scalaire nous 
rappelle que c'est la ville de Troyes qui joue le rôle de référent identitaire puisque tant bien même l'instrumentalisation du lieu a supposé des déplacements dans l'agglomération, c'est bien cette dernière qui véhicule l'image globale du site.

Il en résulte que les concentrations créées réutilisent ces référents patrimoniaux, bien qu'il s'agisse de créations nouvelles. C'est le cas du centre de magasins d'usine Mc Arthur Glen sur la commune de Pont-Sainte-Marie, dans la périphérie de Troyes. Les choix architecturaux (usage de la brique sans parcimonie, création de halls de vente censés rappeler des entrepôts, etc.) s'inscrivent dans cette logique, reprenant ainsi les codes véhiculés par la chaîne de distribution qui font le succès des centres de seconde génération. Le centre de magasins d'usine implanté à Pont-Sainte-Marie, est ainsi comparable par son traitement à ses équivalents britanniques.

En revanche, tous les autres magasins d'usine qui se sont développés autour du Mc Arthur Glen par contagion (Laulajainen, 1991) ou métamorphisme de contact (Reynaud, 1981), ne répondent pas à ces critères de marketing. Ils reprennent qualités et défauts des centres de première génération (mélange avec d'autres activités marchandes notamment), mais s'appuient sur l'image du centre de seconde génération pour assurer leur pérennité. Ils s'inscrivent ainsi dans une relation de commensalisme marchand avec l'entité attractive (Lebrun, 2001).

Figure 2 : Plan de la ZAC de Pont-Sainte-Marie dans l'agglomération de Troyes

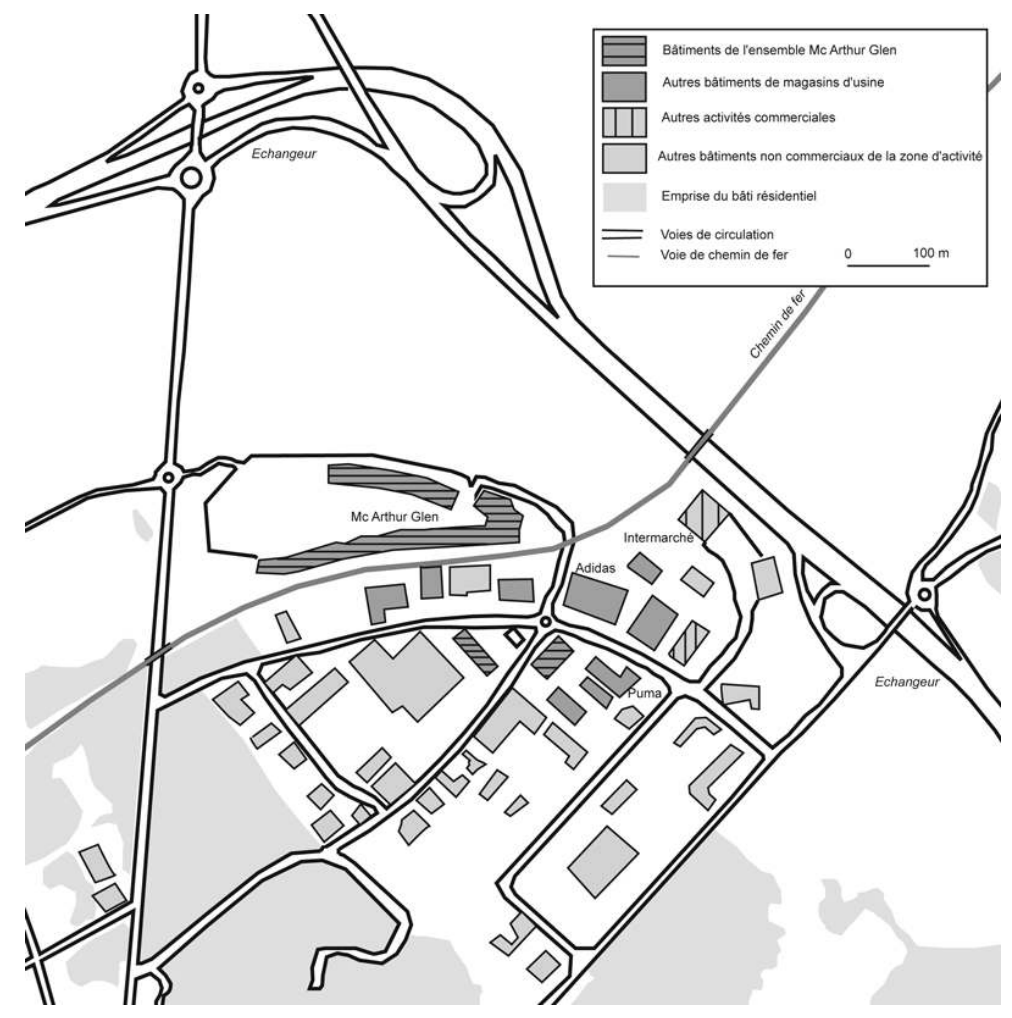

Ainsi, si ces deux espaces commerciaux sont de purs produits de marketing urbain sans lien direct avec un quelconque passé industriel, l'attachement à l'image textile de l'agglomération troyenne nous rappelle que ces deux concentrations n'auraient pu voir le jour ailleurs. Si Troyes possède ces deux centres surdimensionnés pour une agglomération de 120000 habitants, c'est parce que la clientèle consent, pour s'y rendre, à des déplacements bien plus significatifs que pour n'importe quel centre 
commercial régional généraliste. Mais, là encore, comme pour l'Usine à Roubaix, si la patrimonialisation est effective elle n'a que des retombées économiques restreintes sur les autres fonctions urbaines et une portée en termes d'aménagement urbain limité.

\section{Le commerce, instrument de réhabilitation ou de renouvellement urbain : l'exemple de l'aménagement du centre de Roubaix}

Pour Roubaix comme pour Troyes, nous devons constater que l'impact en terme de renouvellement et de réhabilitation urbains est somme toute faible. En effet, il n'affecte qu'un espace restreint, qui plus est périphérique, dans une ancienne friche industrielle, sans entraîner de réelle redynamisation des espaces urbains environnants.

Pourtant, inscrit dans une réflexion d'urbanisme plus globale, l'outil marchand peut intégrer des réflexions plus lourdes quant au devenir de l'urbain. La restructuration du centre roubaisien, au tournant des années 2000 s'inscrit pleinement dans une double logique :

1. Une logique de renforcement de la centralité du centre-ville roubaisien. Cela a pu se faire en le dotant d'équipements généralistes suffisants pour arrêter sa désaffection au profit des centralités périphériques.

2. Une logique de développement d'un pôle marchand spécialisé d'agglomération, se reposant sur l'image véhiculée par le passé urbain et susceptible de drainer une clientèle de toute l'agglomération Lille-Roubaix-Tourcoing et de son aire de chalandise.

La première logique est à l'origine de l'installation d'un centre commercial intégré de centre-ville de taille moyenne (Espace Grand Rue, avec un hypermarché Géant pour locomotive) et d'un cinéma multiplexe. La seconde logique est à l'origine de l'ouverture, à quelques mètres de là, d'un espace marchand piétonnier occupé par un centre de magasins d'usine Mc Arthur Glen. Cet équipement, situé à l'emplacement d'un ancien centre commercial vétuste et déserté, prend la forme d'un mail piétonnier à ciel ouvert pour ne pas introduire de rupture entre le centre-ville et le faubourg de Lannoy au sud de la commune.

34 A la jonction entre ces deux pans du «nouveau » centre-ville roubaisien se situe un nœud de transport important constitué d'un accès au métro, au tramway et aux bus d'une part, et, pour les automobilistes, d'axes pénétrants et d'une offre de stationnement renforcée d'autre part. Ce dispositif lourd a bien sûr été tout autant marqué par l'arrivée du métro, en 2000, que par le seul renforcement de l'offre commerciale. Cette diversité recréée, accompagnée d'un effort de rénovation urbaine, permet à la municipalité d'envisager de faire renaître un centre-ville devenu moribond. Il doit être capable d'éviter la fuite vers les centres commerciaux périphériques ou vers Lille d'une partie des Roubaisiens, tout en donnant des raisons de venir à d'autres habitants de Lille Métropole. (Lebrun, 2004) 
Photo 2 : Le centre commercial Mc Arthur Glen de Roubaix à son ouverture

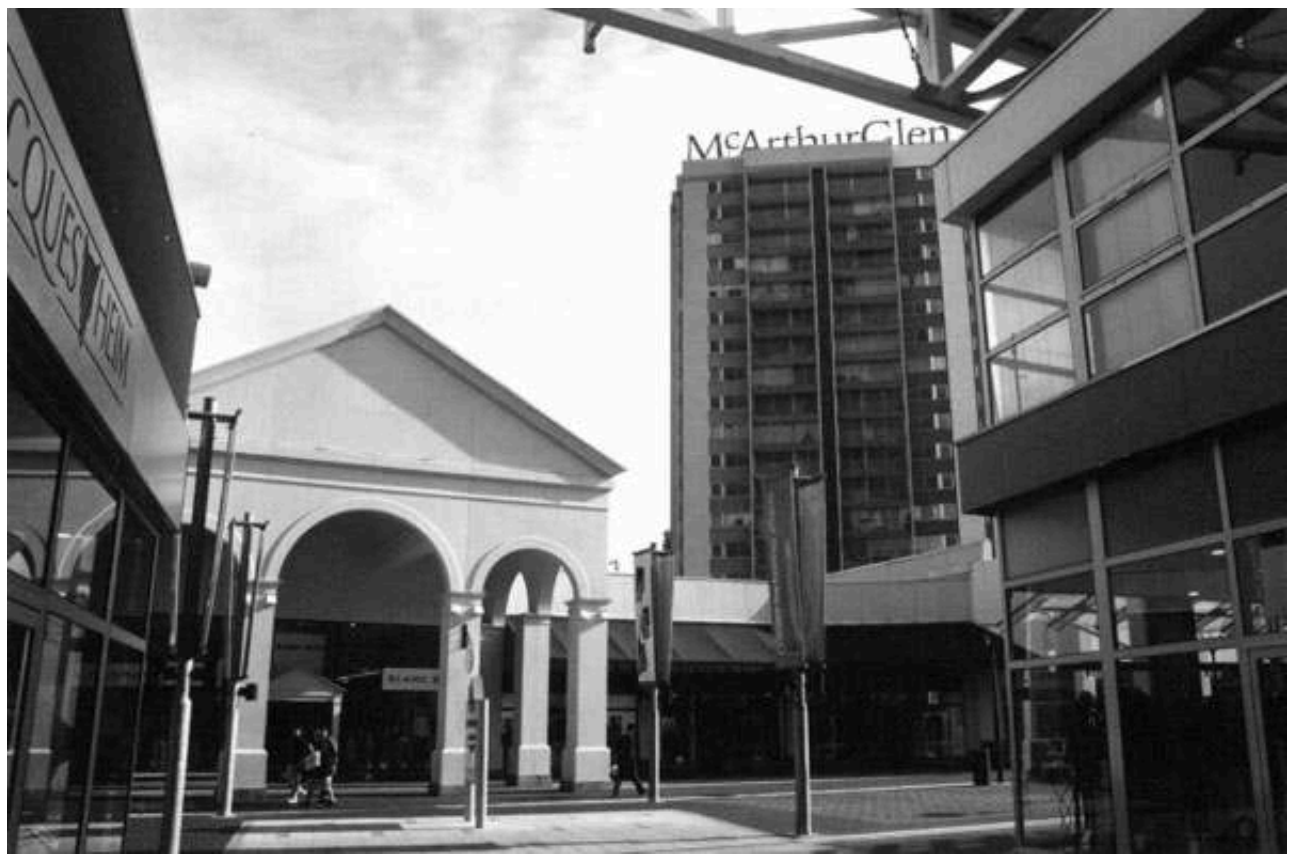

N. Lebrun, 2000

On remarque alors que l'implantation de Mc Arthur Glen Roubaix répond à des normes d'implantation différentes de celle de Mc Arthur Glen Troyes :

- L'implantation roubaisienne a d'emblée été pensée comme une partie d'un projet d'urbanisme global. C'est donc le centre-ville tout entier qui a vocation à constituer un pôle attractif, alors que dans le cas troyen le centre commercial, isolé, ne peut compter que sur sa propre notoriété. C'est ce qui explique que les concepteurs roubaisiens ont pu se permettre d'opter pour un ensemble de boutiques de taille relativement modeste (une quarantaine à l'ouverture en 2000) alors qu'à Troyes l'implantation périphérique fait de la concentration marchande un pôle monofonctionnel. La configuration spatiale d'un centre-ville ne permet pas un aménagement plus conséquent et empêche une évolution ultérieure: le centre devait constituer une entité cohérente, suffisante et viable dès son ouverture.

37 - La forme prise par le centre roubaisien est inédite puisque les centres de magasins d'usine à cette enseigne sont tous des centres commerciaux périphériques bien reliés aux réseaux de transport mais sans aucun lien avec le tissu urbain adjacent. Ici, nous sommes en présence d'un espace marchand privé qui a vocation à servir de trait d'union urbain entre deux composantes de la ville: le centre-ville et le faubourg de Lannoy adjacent. Le traitement en rue piétonne de l'ensemble va en ce sens. Pourtant, une fois les boutiques fermées, la «rue » est clôturée à chacune de ses extrémités, la temporalité du lien retrouvé entre le centre-ville et les quartiers devant se confondre avec la temporalité imposée par la fonction commerciale. Ce décrochage entre le souci de la municipalité et le souci du promoteur marchand souligne l'artificialité et la rareté d'une telle démarche de coopération. Mais si le décollage économique de Mc Arthur Glen Roubaix a été lent, c'est d'abord en raison du décalage de l'offre par rapport au site d'implantation (visant une clientèle plutôt aisée) plus qu'en raison d'erreurs éventuelles d'aménagement. 


\section{Conclusion} était à l'origine même de l'émergence du commerce d'usine dans ces espaces. Et aujourd'hui, c'est le commerce qui permet, sinon de maintenir l'industrie, du moins de redonner une dimension économique et sociale à certains espaces de friches.

\section{BIBLIOGRAPHIE}

AUGE M. (1991). - « Le patrimoine contre la mort ». In : Dulau R., Apologie du périssable, édition du Rouergue, p. 331-333.

Bondue J-P. (2000) - « La nouvelle armature commerciale du Nord-Pas de Calais », Hommes et Terres du Nord, $\mathrm{n}^{\circ} 2000 / 1$, p. I-IV

Collectif (2003) - Analyse et développement du concept de magasins d'usine et de négoce. Situation comparée des centres de Troyes, Roubaix et Talange, Troyes, CCI Troyes et Aube, $126 \mathrm{p}$.

Collectif (2004) - Centres de marques. Les nouvelles approches du concept en Europe, Troyes, CCI Troyes et Aube, $17 \mathrm{p}$.

DevisMe L. (1998). - Actualité de la pensée d'Henri Lefebvre à propos de l'urbain. La question de la centralité, Tours, Travaux de la Maison des Sciences de la Ville, $126 \mathrm{p}$.

FOUCART B. (2003). - " Accepter la pluralité des réponses », Lettre d'information du Ministère de la Culture et de la Communication, Paris, $\mathrm{n}^{\circ} 111$, p. 8 
GRAVARI-BARBAS M. (1996). - « Le patrimoine, facteur d'appartenance à un territoire urbain », Géographie et Cultures, ${ }^{\circ}$ 20, p. 55-68

GREFFE X. (2003) - La valorisation économique du patrimoine, Paris, La Documentation Française, $384 \mathrm{p}$.

LEBRUN N. (2001). - «Vers une approche des types de liens entre accessibilité et commerce », Travaux de l'Institut de Géographie de Reims, Reims, n 107-108, p. 25-40

LEBRUN N. (2002). - Centralités urbaines et concentrations de commerces, Reims, thèse de doctorat, $513 \mathrm{p}$.

LEBRUN N. (2004). - « La revitalisation du centre de Roubaix entre centre-ville de proximité et pôle spécialisé d'agglomération ». In : LESTRADE S., Commerce et requalification des territoires sensibles. Les nouvelles dynamiques commerciales en milieu urbain et rural, Créteil, CERAC - Univ. Paris XII, p. $117-128$

LAULAJAINEN R. (1991). - « Two retailers go global : the geographical dimension ", The International Review of Retail, Distribution and Consumer Research, Londres, Routledge, p. 607-626.

MoATI P. (2001) - L'avenir de la grande distribution, Paris, Odile Jacob, 392 p.

PARIS D. (2001) - «Renouveler la ville : un enjeu de développement pour la ville postindustrielle ». In : PARIS D. et ROBERT J., Renouveler la ville. Les enjeux de la régénération urbaine, Lille, FREVille, p. 8-15

ReYNAUd A. (1981) - Société, espace et justice, Paris, P.U.F., 263 p.

\section{RÉSUMÉS}

La crise du secteur industriel provoque des évolutions des formes urbaines. Les friches doivent être réappropriées. La fonction commerciale est souvent utilisée pour réinvestir ces lieux. Mais seuls les magasins d'usine permettent de vraiment garder un lien avec les activités passées du lieu.

The industrial crisis provokes evolution of urban forms. Some abandoned areas must be transformed. The commercial function is frequently used to reinvigorate these places. However, only factory warehouses are permitted to properly retain a link with the past activities of the place.

Die Krise des Industriesektors ruft Veränderungen des Stadtbildes und der städtischen Strukturen hervor, und es gilt, die Industriebrachen einer neuen Nutzung zuzuführen. Häufig investiert dabei der Handel in solche Standorte. Jedoch kann lediglich der Fabrikverkauf (Factory Outlet Center) wirklich eine Bindung mit den ehemaligen Aktivitäten bewahren.

\section{INDEX}

Keywords : warehouses, heritage, conversion, urban space

Mots-clés : magasin d'usine, patrimoine, reconversion, espace urbain

Schlüsselwörter : Factory Outlet Center, Erbe, Wiederaufbau, Stadterneuerung 


\section{AUTEUR}

\section{NICOLAS LEBRUN}

Docteur en Géographie et Aménagement/Professeur du secondaire - Lycée Saint-Rémi - 6, place Winston Churchill 08000 Charleville-Mézières - lebrunni@hotmail.fr 